with representative Japanese data report from National Health and Nutrition Survey.

Results 243 participants, 117 males and 126 females, completed questionnaire and measurements. Elderly people aged 65 and over were $58.2 \%$ in males and $61.9 \%$ in females. Prevalence (\%) of overweight, BMI>25.0, were $36.3 / 24$. for males/females, whereas 28.6 $/ 20.6$ in Japan. Waist circumference more than $85 \mathrm{~cm}$ for male and $90 \mathrm{~cm}$ for female, were 80.2/38.4 whereas 52.9/17.9 in Japan. Hypertension, SBP $>140$ and/or DBP $>90$, were 60.9/57.4, whereas 42.8/31.5 in Japan. Probable diabetes, FBS $>126 \mathrm{mg} / \mathrm{dl}$, and high triglyceride, $>150 \mathrm{mg} / \mathrm{dl}$, were almost same. Metabolic syndrome were noted 41.8/12.6 whereas 25.3/10.6 in Japan.

Conclusion High prevalence of MetS among Japanese Immigrant Population with big dietary change in South Brazil was indicated.

\section{P2-195 A PROPENSITY SCORE FOR PREDICTING MAJOR ADVERSE OUTCOMES AFTER TOTAL JOINT REPLACEMENT IN MEN}

doi:10.1136/jech.2011.142976j.29

${ }^{1} \mathrm{G}$ Mnatzaganian, ${ }^{* 1,2} \mathrm{P}$ Ryan, ${ }^{3} \mathrm{P}$ Norman, ${ }^{4} \mathrm{D}$ Davidson, ${ }^{1,5} \mathrm{~J}$ Hiller. ${ }^{1}$ Department of Public Health, The University Of Adelaide, Adelaide, South Australia, Australia; ${ }^{2}$ Data Management and Analysis Centre, the University of Adelaide, Adelaide, South Australia, Australia; ${ }^{3}$ School of Surgery, University of Western Australia, Perth, Western Australia, Australia; ${ }^{4}$ Royal Adelaide Hospital, Adelaide, South Australia, Australia; ${ }^{5}$ Faculty of Health Science, Australian Catholic University, Victoria, Australia

Objective Prioritisation of patients for total joint replacement (TJR) represents a challenge. The objective of this study was to construct a propensity score (PS) that both predicts and informs prioritisation of TJR among elderly men.

Methods Clinical data from 11388 men were integrated with hospital morbidity data and mortality records. A PS quantifying each individual's probability of having TJR was calculated using multivariable competing risk regression models. The PS was then used to assess risk of incident in-hospital complications and mortality following TJR.

Results Younger and healthier patients were selected for TJR. Comorbidities such as diabetes mellitus, peripheral vascular disorders, and cancer lowered the probability of having TJR. Among men who had TJR, 25\% developed a major in-hospital complication. The PS independently predicted both major complications and short- and long-term mortality. Patients with low PSs who nonetheless underwent TJR were more likely to experience an adverse outcome. After adjusting for risk factors, patients who were in the lowest tertile of the distribution of the PS were $67 \%$ more likely to develop a major complication $(p=0.023)$, and 2.2 times more likely to die within 10 years after TJR $(p=0.035)$. Other predictors of major complications following TJR included weight, injury, and having a minor complication. In-hospital complications independently increased the risk of mortality after TJR.

Conclusion In the presence of clinical indications for TJR, this PS informs clinical decision making about selecting patients who are most likely to benefit and least likely to be harmed as a consequence of TJR.

\section{P2-196 SMOKING, BODY WEIGHT, PHYSICAL EXERCISE AND RISK OF LOWER LIMB TOTAL JOINT REPLACEMENT IN A POPULATION-BASED COHORT OF MEN}

doi:10.1136/jech.2011.142976j.30

${ }^{1} \mathrm{G}$ Mnatzaganian, ${ }^{* 1,2} \mathrm{P}$ Ryan, ${ }^{3} \mathrm{P}$ Norman, ${ }^{4} \mathrm{D}$ Davidson, ${ }^{1,5} \mathrm{~J}$ Hiller. ${ }^{1}$ The University of Adelaide, Adelaide, South Australia, Australia; ${ }^{2}$ Data Management and Analysis Centre, The University of Adelaide, Adelaide, South Australia, Australia; ${ }^{3}$ School of Surgery, University of Western Australia, Perth, Western Australia, Australia; ${ }^{4}$ Royal
Adelaide Hospital, Adelaide, South Australia, Australia; ${ }^{5}$ Faculty of Health Science Australian Catholic University, Victoria, Australia

Objective To assess the associations of smoking, body weight and physical activity with the risk of undergoing total joint replacement (TJR) in a population-based cohort of men.

Methods A cohort study of 11388 men that integrated clinical data with hospital morbidity data and mortality records. In three separate age groups we modelled the risk of TJR on weight, height, comorbidity, injury, socioeconomic status, years of smoking and exercise, using Cox proportional hazards regressions and competing risk regressions.

Results A dose-response relationship between both weight and smoking, and risk of TJR was observed. Being overweight independently increased the risk of TJR, while smoking lowered the risk. The decreased risk among smokers was demonstrated in both Cox and CRR models and it became apparent after 23 years of exposure. Men who were in the highest quartile (48+ years of smoking) were $44 \%-52 \%$ less likely to undergo TJR than never-smokers. Tests for trend in the log HRs across both smoking and weight quantiles yielded $\mathrm{p}<0.001$. Vigorous exercise increased the hazard of TJR, however, the association reached statistical significance only in the 70-74 year-old age-group (adjusted-hazard ratio: $1.71,95 \%$ CI 1.26 to 2.33). Adjusting for Deyo-Charlson Index or Elixhauser's comorbidities did not eliminate these associations.

Conclusion Being overweight and reporting vigorous physical activity increased the risk of TJR. This study is the first to demonstrate a strong inverse dose-response effect of duration of smoking and TJR. More research is needed to better understand the role of smoking in the pathogenesis of osteoarthritis.

\section{P2-197 CASE-CONTROL ANALYSIS OF THE EFFECTS OF AGE AND GEOHELMINTH INFECTION ON WHEEZE AND ATOPY IN THE RURAL TROPICS}

doi:10.1136/jech.2011.142976j.31

${ }^{1,2} \mathrm{~A}$ L Moncayo, ${ }^{* 1} \mathrm{M}$ Vaca, ${ }^{1} \mathrm{~S}$ Erazo, ${ }^{1} \mathrm{G}$ Oviedo, ${ }^{1}$ O Quinzo, ${ }^{1} \mathrm{M}$ E Chico, ${ }^{3} \mathrm{~T}$ A E PlattsMills, ${ }^{4} \mathrm{~L}$ C Rodrigues, ${ }^{2} \mathrm{M}$ L Barreto, ${ }^{1,5} \mathrm{P} \mathrm{J}$ Cooper. ${ }^{1}$ Universidad San Francisco de Quito, Colegio de Ciencias de la Salud, Quito, Ecuador; ${ }^{2}$ Universidade Federal da Bahia, Instituto de Saúde Coletiva, Salvador, Bahia, Brazil; ${ }^{3}$ University of Virginia, Asthma and Allergic Diseases Center, Virginia, USA; ${ }^{4}$ London School of Hygiene and Tropical Medicine, Department of Epidemiology, London, UK; ${ }^{5}$ Liverpool School of Tropical Medicine, Liverpool, UK

Introduction Epidemiological data suggest that a minority of asthma cases in Latin America may be associated with allergic sensitisation which could be explained by the effect of environmental factors. The aim was to explore the relationship between levels of $\operatorname{IgE}$ specific for allergens (asIgE), skin prick test responses (SPT) and recent wheeze and the effect of age and geohelminth infections on these associations.

Methods A case-control study was conducted among 376 children aged $7-19$ yrs living in Afro-Ecuadorian rural communities in tropical Ecuador. Asthma cases were selected based on the presence of recent wheeze and controls as a random sample of those without symptoms by questionnaire. Atopy was measured either by asIgE or SPT. Geohelminth infection was determined by both eggs in stools and anti-Ascaris IgE.

Results There was a significant positive association between the markers of atopy and recent wheeze in the older (7-11 yrs) but not in the younger (12-19yrs) age group. A positive association between anti-Ascaris IgE and wheeze was observed in both the younger (adj. OR 2.06, 95\% CI 1.05 to 4.03 ) and the older age groups (adj. OR 3.00, 95\% CI 1.37 to 6.56). Having SPT responses was significantly associated with wheeze among children with no active geohelminth infection (adj. OR 3.52, 95\% CI 1.05 to 11.79), while 
this association was lost among children with active infection (adj. OR $1.59,95 \%$ CI 0.72 to 3.54 ).

Conclusions The association between markers of atopy and wheeze increased with age. The results suggest modification of the relationship between SPT and wheeze by active geohelminth infections.

\section{P2-198 OVERWEIGHT ASSOCIATED WITH NON-ATOPIC WHEEZE IN RURAL TROPICS}

doi:10.1136/jech.2011.142976j.32

\begin{abstract}
${ }^{1,2} \mathrm{~A}$ L Moncayo, ${ }^{*}{ }^{1} \mathrm{M}$ Vaca, ${ }^{1} \mathrm{~S}$ Erazo, ${ }^{1} \mathrm{G}$ Oviedo, ${ }^{1} \mathrm{I}$ Quinzo, ${ }^{1} \mathrm{M}$ E Chico, ${ }^{3} \mathrm{~T}$ A E PlattsMills, ${ }^{2} \mathrm{~S}$ Alvim, ${ }^{4} \mathrm{~L}$ C Rodrigues, ${ }^{2} \mathrm{M}$ L Barreto, ${ }^{1,5} \mathrm{P}$ J Cooper. ${ }^{1}$ Universidad San Francisco de Quito, Colegio de Ciencias de la Salud, Quito, Ecuador; ${ }^{2}$ Universidade Federal da Bahia, Instituto de Saúde Coletiva, Salvador, Bahia, Brazil; ${ }^{3}$ University of Virginia, Asthma and Allergic Diseases Center, Virginia, USA; ${ }^{4}$ London School of Hygiene and Tropical Medicine, Department of Epidemiology, London, UK; ${ }^{5}$ Liverpool School of Tropical Medicine, Liverpool, UK
\end{abstract}

Introduction The parallel rise in prevalence of asthma and overweight/obesity in some Latin American countries has led to suggestions of a link between the two epidemics. The aim of this study was to explore the effects of being overweight on wheeze, exercise-induced bronchospasm and atopy.

Methods A case-control study was conducted among 809 AfroEcuadorian children aged 7-19yrs living in rural communities in tropical Ecuador. Asthma cases were selected based on the presence of recent wheeze and controls as a random sample of those without symptoms by questionnaire. Atopy was measured either by the presence of allergen specific IgE (asIgE) in serum or by allergen skin test reactivity (SPT). Overweight children were those with a Body Mass Index (BMI) $\geq+1$ z-score based on WHO growth curves (2007). Results Comparing atopic vs non-atopic children, the prevalence of SPT (adj. OR 2.12, 95\% CI 1.22 to 3.68) and the presence of asIgE (adj. OR 2.30, 95\% CI 1.10 to 4.83) was greater in overweight children compared to those with normal weight/underweight. Comparing non-atopic wheezers with non-atopic non-wheezers, being overweight was significantly associated with non-atopic wheeze (adj. OR 2.22, 95\% CI 1.07 to 4.63) when atopy was defined as asIgE but not SPT. Being overweight was not significantly associated with atopic wheeze (comparing atopic wheezers with atopic non-wheezers) and with severe wheeze or exercise-induced bronchospasm irrespective of atopic status.

Conclusion Although being overweight was associated with atopy, it was also associated with wheeze, and somewhat surprisingly this latter effect was not observed among atopic children.

\section{P2-199 ASSOCIATION BETWEEN ADHERENCE TO THE MEDITERRANEAN DIET AND BONE QUALITY IN A SAMPLE OF PORTUGUESE ADOLESCENTS}

doi:10.1136/jech.2011.142976j.33

\author{
${ }^{1,2} \mathrm{~T}$ Monjardino, ${ }^{* 1,2} \mathrm{R}$ Lucas, ${ }^{1,2}$ E Ramos, ${ }^{1,2} \mathrm{H}$ Barros. Institute of Public Health of the \\ University of Porto, Porto, Portugal; ${ }^{2}$ University of Porto Medical School, Porto, Portugal
}

Introduction Dietary patterns provide insights into how diet, rather than specific nutrients, affects bone health. We aimed to evaluate whether Mediterranean diet associates with bone mineral density at 13 years-old.

Methods We used data from 1232 adolescents (44.7\% males) born in 1990 and assessed at 13 years-old (EPITeen cohort). Adolescents were evaluated through physical examination, including height, weight and forearm bone mineral density (BMD) using dual-energy x-ray absorptiometry. Dietary intake was assessed using a food frequency questionnaire and adherence to the Mediterranean diet was evaluated through an adapted score (KIDMED index). The final score, the sum of all items, was classified into three adherence levels: -2 to 3,4 to 6 and $\geq 7$. The association between KIDMED index and $\mathrm{BMD}$ was quantified using linear regression. Coefficients were adjusted for body mass index, physical activity, smoking status and parental formal education.

Results Low KIDMED index was found in $23.9 \%$ of the girls and in $22.5 \%$ of the boys and $47.3 \%$ of girls and $46.1 \%$ of boys had intermediate index results. Mean (SD) BMD was $0.361(0.058) \mathrm{g} / \mathrm{cm}^{2}$ in girls and $0.344(0.051) \mathrm{g} / \mathrm{cm}^{2}$ in boys. Adherence to the Mediterranean diet showed no relation with $\mathrm{BMD}$ in girls. Significantly higher average $\mathrm{BMD}$ was found among boys with intermediate (0.013, 95\% CI: 0.003 to 0.023$)$ and high KIDMED index (0.017, 95\% CI 0.006 to 0.028 ) when compared to those with low index.

Conclusion A Mediterranean dietary pattern can be associated to better bone health since early in life.

\section{P2-200 POPULATION TRENDS IN THE INCIDENCE AND OUTCOMES OF ACUTE MYOCARDIAL INFARCTION IN A MEDITERRANEAN REGION OF SOUTHERN EUROPE}

doi:10.1136/jech.2011.142976j.33a

${ }^{1} \mathrm{C}$ Moreno-lribas, ${ }^{2} \mathrm{M}$ Imizcoz, ${ }^{1} \mathrm{M}$ Guevara, ${ }^{1} \mathrm{~J}$ Delfrade, ${ }^{3} \mathrm{~N}$ Alvarez-Arruti. ${ }^{*}$ Public Health Institute of Navarra, Pamplona-Iruña, Spain; ${ }^{2}$ Hospital Complex of Navarra, Pamplona-Iruña, Spain; ${ }^{3}$ Navarra Health Service, Pamplona-Iruña, Spain

Introduction The aim of this population-based study was to examine trends in the myocardial infarction incidence and mortality rates between 2000 and 2009 in Navarre, Spain.

Methods All admissions for myocardial infarction in a region of over half a million inhabitants from January 2000 to December 2009 were identified from the hospitals discharge databases. Age- and sexadjusted incidence and 30-day and 30-365-day mortality rates were calculated.

Results We identified 4451 persons 30 years of age or older with a first myocardial infarction. The incidence showed a significant decrease from 128 cases per 100000 person-years in 2000 to 98 cases per 100000 person-years in 2009, a 23\% relative decrease. Only in the case of ST-segment elevation myocardial infarction the decrease was significant. The proportion of patients who underwent revascularisation within 30 days after myocardial infarction increased from $26.8 \%$ in 2000 to $65.6 \%$ in 2009 . The age- and sex-adjusted 30 day mortality after myocardial infarction decreased from $10.7 \%$ in 2000-2004 to $7.6 \%$ in $2005-2009$. No changes were observed in 30-365-day mortality.

Discussion The important fall in the incidence observed in this population is consistent with other studies from other industrialised areas of the World. The higher rates of ST-segment elevation myocardial infarction vs non-ST could be indicating that the new definition is not broadly applied. The decreasing incidence, particularly ST-segment elevation myocardial infarction, is probably explained, at least in part, by substantial improvements in primary prevention efforts. Important improvements were observed also in the hospital care, particularly the big increase in revascularisation.

\section{P2-201 CHANGES IN AMINOTRANSFERASES INDICATE CHANGES IN HEPATOSTEATOSIS IN PEOPLE WITH TYPE 2 DIABETES: THE EDINBURGH TYPE 2 DIABETES STUDY}

doi:10.1136/jech.2011.142976j.34

${ }^{1} \mathrm{~J}$ Morling, ${ }^{*}{ }^{2} \mathrm{R}$ Williamson, ${ }^{2} \mathrm{M}$ Strachan, ${ }^{1} \mathrm{~J}$ Price, ${ }^{2} \mathrm{~S}$ Glancy, ${ }^{2} \mathrm{~L}$ Nee. ${ }^{1}$ University of Edinburgh, Edinburgh, UK; ${ }^{2}$ Western General Hospital, Edinburgh, UK

Background Recent data have suggested that plasma aminotransferases may be of limited use in the diagnosis of non-alcoholic 